\title{
Photometry of CH Cygni During 1991-1994 ${ }^{\star}$
}

\author{
K.P. Panov ${ }^{1}$, J.S.W. Stegert ${ }^{2}$, G. Hildebrandt ${ }^{3}$ \\ 1 Institute of Astronomy, Bulgarian Academy of Sciences, Zarigradsko Chaussee 72, \\ 1784 Sofia, Bulgaria \\ 2 Sternwarte der Universität Bonn, Auf dem Hügel 71, 53121 Bonn, Germany \\ 3 Astrophysikalisches Institut Potsdam, An der Sternwarte 16, 14482 Potsdam, \\ Germany
}

$\mathrm{CH}$ Cygni has been observed since about one decade at the Bulgarian National Astronomical Observatory Rozhen. $U B V$ photometry was obtained with the $60 \mathrm{~cm}$ telescope. The computer controlled photometer is equipped with an EMI 9789 QB multiplier, a set of $u, b, v$ filters and a diaphragm of $28^{\prime \prime}$. The reduction includes corrections for dead-time and background, differential extinction and transformation to the standard $U B V$ system.

Observations between 1991 and 1994 are displayed in Fig. 1 and show the new outburst, which is still going on. The previous major outburst lasted until 1984, and CH Cyg declined slowly between 1985 and 1988. In 1988, it reached its minimum in brightness and activity. The cause for the activity is probably mass transfer from the $\mathrm{M}$ giant component onto the hot companion. In this model, only the $M$ star is seen during activity minimum. In July 1988, the mean brightness was $V=8.50, B-V=1.39$ and $U-B=1.48$. Since 1989, some erratic activities appeared, including a hot variable continuum, broad and variable emissions in the Balmer lines and rapid light variations (Leedjarv 1990, Tomov and Mikolajewski 1992, Kuczawska et al. 1992, Panov and Ivanova 1992). Since 1991, a trend of increasing activity is observed, which persisted also in 1994. The brightening of $\mathrm{CH}$ Cyg of about $5^{\mathrm{m}}$ in $U$ in 1994 with respect to 1991 shows that it undergoes another major outburst. Moreover, it exhibits light variations with different other time scales, minutes (flickering), days and weeks, probably resulting from the mass-transfer. Fig. 2 showns an example of a short time variation on October 1, 1994. The flickering of $\mathrm{CH} C \mathrm{Cyg}$ was very pronounced in the $U, B$ and $V$ filters and the amplitudes reached several $0^{\mathrm{m}} 1 \mathrm{in}$ about half an hour. More observations of this interesting system are planned.

\section{References}

Kuczawska E., Mikolajewski M., Kirejczyk K., 1992, IBVS 3806

Leedjarv L., 1990, IBVS 3474

Panov K.P., Ivanova M.S., 1992, IBVS 3817

Tomov T., Mikolajewski M., 1992, IBVS 3721

\footnotetext{
^ Based on observations collected at the National Observatory, Rozhen, Bulgaria
} 

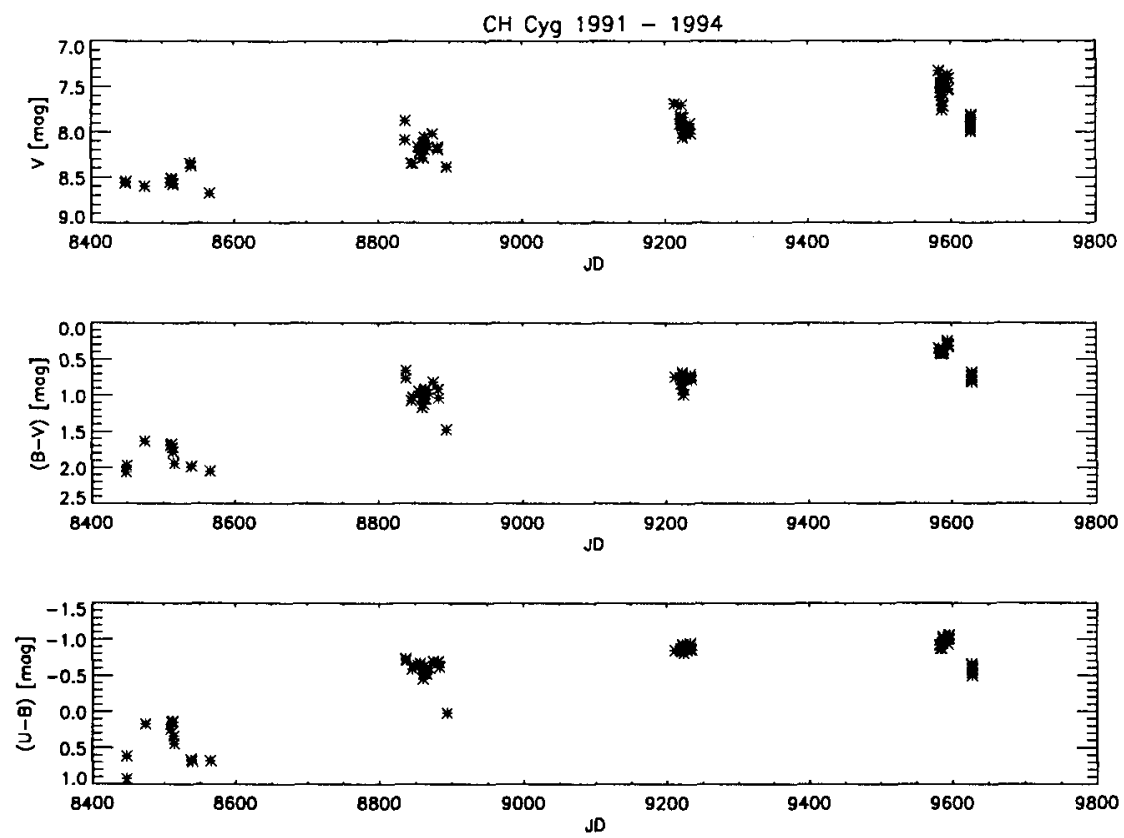

Fig. 1. UBV photometry of CH Cygni between 1991 and 1994
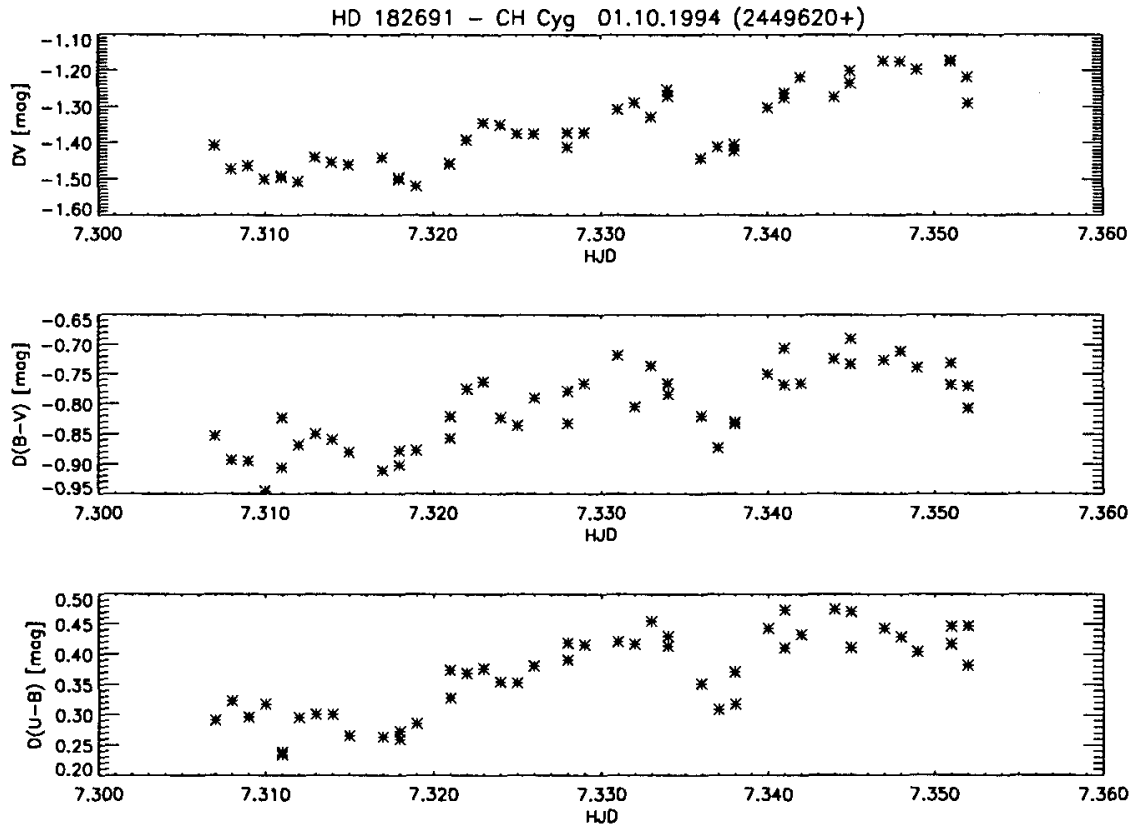

Fig. 2. Short time light variations of $\mathrm{CH}$ Cygni on October 1, 1994. 